\title{
Social Media, Research, and Ethics: Does Participant Willingness Matter?
}

\section{Findings from the 2020 International Medical Informatics Association (IMIA) Yearbook of Medical Informatics, Section on Consumer Health Informatics and Education}

\author{
Pascal Staccini', Annie Y. S. Lau' ${ }^{2}$, Section Editors for the IMIA Yearbook Section on Consumer \\ Health Informatics and Education \\ I IRIS Department, Lab RETINES, Faculté de Médecine, Université Côte d'Azur, France \\ ${ }^{2}$ Centre for Health Informatics, Australian Institute of Health Innovation, Macquarie University, Australia
}

\section{Summary}

Objective: To summarise the state of the art published in 2019 in consumer health informatics and education, with a special emphasis on "Ethics and Health Informatics".

Methods: We conducted a systematic search of articles published in PubMed using a predefined set of queries, which identified 368 potential articles for review. These articles were screened according to topic relevance and 15 were selected for consideration of best paper candidates, which were then presented to a panel of international experts for full paper review and scoring. The top five papers according to the external reviewers' ranking were discussed in a consensus meeting. Finally, the paper that received the highest score from four of the five experts was selected as the best paper on social media and ethics for patients and consumers of the year 2019.
Results: Despite using the terms "ethics" and "ethical" in the search query, we retrieved very few articles. The bibliometric analysis identified three major clusters centred on "social", "health", and "study". Among the top five papers, one was a review where the authors identified ethical issues across four areas at the intersection of social media and health: 1) the impact of social networking sites on the doctor-patient relationship; 2) the development of e-health platforms to deliver care; 3) the use of online data and algorithms to inform health research; and 4) the broader public health consequences of widespread social media use. The other papers highlighted ethical concerns in using social media to interact with patients at different phases of a clinical research protocol, such as recruitment phase, participant engagement, data linkage, and detection and monitoring of adverse events.
Conclusions: Findings suggest that most users do not think that using social media for patient monitoring in clinical research, for example using Twitter for clinical trial recruitment, constitutes inappropriate surveillance or a violation of privacy. However, further research is needed to identify whether and how views on ethical concerns differed between social media platforms and across populations.

\section{Keywords}

Social media, ethics, research design, privacy, patient selection, bibliometrics

Yearb Med Inform 2020:176-83 http://dx.doi.org/10.1055/s-0040-1702022

\section{Introduction}

For this $29^{\text {th }}$ edition of the Yearbook of Medical Informatics, the special topic of "Ethics and Health Informatics" comes at the time when individuals are increasingly exposed to data sharing practices and growing numbers of researchers and healthcare professionals reuse encoded medical data and social media data to add real-life context to clinical research purposes.

The idea of utilizing social media in clinical research is not recent. From a research perspective, social media is often perceived as a tool to facilitate patient recruitment, monitor patient involvement, and improve patient retention in clinical trials $[1,2]$. The ubiquitous use of online social platforms has also led researchers to envision new ways in which social media can promote better health outcomes. There is growing interest in building a "social mediome" using data derived from social media platforms that reveal individual- and population-level health information to gain greater insight into patient health habits to facilitate treatment [3].

The special topic of the 2020 Yearbook of Medical Informatics is timely since social media data collected by devices and web applications are increasing exponentially. Yet the ethical concerns to use such data for research purposes warrant further consideration, as the rigorous ethical principles applied in clinical research are often not transferred into the online and social media world [4].

But those massive amounts and varying types of data are attractive not only to traditional researchers at academic institutions, but also to non-traditional researchers, such as commercial entities and citizen or community scientists. Devices, web applications, and social online platforms collect a myriad of data in varying details, where such data is made available to third parties for research and other purposes, many of which may be unregulated, and often without users' awareness or permission. The health-related nature of data collected in such a way may 
affect user perception of and expectation for privacy. Users may expect that their data are protected because data are health-related. This misconception could be compounded if a health care provider recommends or encourages patients to use a device to $\log$ and track symptoms or health behaviours. In any event, patients may reveal personal or sensitive health-related information without realizing that such data are not protected from unwanted use or access, such as targeted advertising.

\section{Methodology}

\section{Search Strategy}

We used PubMed to conduct our search, capturing papers published in the year 2019 on consumer-facing technologies and ethical concerns. The search strategy was based on the PICO framework (P-Population/ Problem, I-Intervention, C-Comparison, O-Outcome), where 'Problem' refers to the various digital environments consumers and patients participate in (e.g., social media, online health communities); "Intervention" is considered as the topic "ethics" (e.g., philosophy, integrity, honour, respect, right); "Outcome" outlines the result of ethical effects in health (e.g., benefit, risk, issue, policy, guidelines). A 'comparison' intervention was not included as it is not relevant in this review. We started from a core query adopted in previous work. Step by step, we refined the query to include keywords related to digital/social media (42 keywords), ethics (23 keywords), and results (5 keywords). MeSH terms and the syntax "[All Fields]" were used wherever possible to ensure our search strategy was comprehensive. The final search query is listed below:

((2019[DP] NOT "Epub ahead of print") NOT Bibliography[pt] NOT Comment[pt] NOT Editorial[pt] NOT Letter[pt] NOT News[pt] NOT Case Reports[pt] NOT Published Erratum[pt] NOT Historical Article[pt] NOT Legal Case[pt] NOT legislation[pt] NOT ("review"[pt] OR "review literature as topic"[MeSH Terms] OR "literature review"[All Fields]))
AND ("ehealth"[All Fields] OR "e-health"[All Fields] OR "tele-health"[All Fields] OR "mhealth"[All Fields] OR "telemedicine"[All Fields] OR "electronic patient-physician communication"[All Fields] OR "electronic medical record"[All Fields] "electronic health record"'[All Fields] OR "personal health record"[All Fields] OR "electronic patient record"[All Fields] OR "online"[All Fields] OR "electronic"[All Fields] OR "information technology"[All Fields] OR "communication technology"[All Fields] OR "mobile"[All Fields] OR "online systems"[All Fields] OR "internet"[All Fields] OR "web"[All Fields] OR "website"[All Fields] OR "patient portal"[All Fields] OR "cell phone"[All Fields])

AND ("social media"[All Fields] OR "facebook"[All Fields] OR "twitter"[All Fields] OR "youtube"[All Fields] OR "instagram"[All Fields] OR "social network site"[All Fields] OR "social web"[All Fields] OR "online social network"[All Fields] OR "social environment"[All Fields] OR "social process"[All Fields] OR "social competition"[All Fields] OR "social norm"[All Fields] OR "social feedback"[All Fields] OR "social influence"[All Fields] OR "social comparison"[All Fields] OR "social network"[All Fields] OR "discussion group"[All Fields] OR "support group"[All Fields] OR "social support"[All Fields] OR "community network"[All Fields] OR "online community"[All Fields])

AND ("access"[All Fields] OR "sharing"[All Fields] OR "share"[All Fields] OR "privacy"[All Fields] OR "protection"[All Fields] OR "informed consent"[All Fields] OR "ethics"[All Fields] OR "bioethics"[All Fields] OR "ethical"[All Fields] OR "moral"[All Fields] OR "principle"[All Fields] OR "value"[All Fields] OR "integrity"[All Fields] OR "honour"'[All Fields] OR "right"[All Fields] OR "responsibility"[All Fields] OR "honesty"[All Fields] OR "conscience"[All Fields] OR "fairness"[All Fields] OR "virtue"[All Fields] OR "philosophy"[All Fields] OR "choice"[All Fields] OR "respect"[All Fields])

AND ("benefit"[All Fields] OR "risk"[All Fields] OR "issue"[All Fields] OR "policy"[All Fields] OR "guideline"[All Fields])

\section{Bibliometrics Analysis}

To understand the state of the literature, we applied various bibliometrics tools onto the original set of articles returned from the search query. The "Bibliometrix" package from $R$ [5] was used on the retrieved articles to report frequency of the 50 most popular words found in all abstracts. We also counted the occurrences of keywords. We analysed keywords and words in titles to uncovers links between concepts through word co-occurrences. Conceptual structure is used to reveal topics covered by scholars and identify what are the most important words. We also study co-word network and draw clusters of words (keywords and titles words). We plot a thematic map to analyse these clusters according to the quadrant in which they are placed: (1) upper-right quadrant: motor-themes; (2) lower-right quadrant: basic themes; (3) lower-left quadrant: emerging or disappearing themes; and (4) upper-left quadrant: very specialized or niche themes.

\section{Results}

\section{State of the Literature}

The search query allowed to selected 368 articles. A descriptive analysis of these articles was conducted, analysing the frequency of keywords, and the frequency of words in titles and abstracts. We used 1,109 distinct keywords, 1,714 distinct words in titles, and 8,147 in abstracts.

Table 1 shows keywords occurrences. As a MeSH qualifier, "ethics" was associated with the following MeSH major terms:

- Automation/ethics

- Automobiles/ethics/legislation \& jurisprudence

- Confidentiality/ethics/standards

- Ergonomy/ethics

- Mental health/ethics/standards

- Mobile applications/ethics/standards

- Physician-patient relations/ethics

- Risk management/ethics/legislation \& jurisprudence

- Telemedicine/ethics/standards 
Staccini et al.

Table 1 Frequency of keywords (reported from most frequent to least frequent)

\begin{tabular}{|c|c|c|c|}
\hline Words & Frequency & Words & Frequency \\
\hline humans & 206 & decision making & 7 \\
\hline female & 134 & intention & 7 \\
\hline male & 115 & mental health & 7 \\
\hline adult & 96 & reproducibility of results & 7 \\
\hline middle aged & 64 & self efficacy & 7 \\
\hline adolescent & 53 & choice behavior & 6 \\
\hline surveys and questionnaires & 52 & cohort studies & 6 \\
\hline young adult & 52 & focus groups & 6 \\
\hline social media & 41 & infant & 6 \\
\hline aged & 37 & interpersonal relations & 6 \\
\hline social support & 36 & motivation & 6 \\
\hline internet & 24 & quality of life & 6 \\
\hline qualitative research & 24 & social media/trends & 6 \\
\hline cross-sectional studies & 22 & socioeconomic factors & 6 \\
\hline risk factors & 18 & attitude to health & 5 \\
\hline child & 16 & awareness & 5 \\
\hline health knowledge attitudes practice & 16 & child preschool & 5 \\
\hline pregnancy & 14 & Europe & 5 \\
\hline social networking & 14 & health behavior & 5 \\
\hline aged 80 and over & 12 & health promotion/methods & 5 \\
\hline communication & 12 & $\begin{array}{l}\text { Homosexuality } \\
\text { male/psychology/statistics \& } \\
\text { numerical data }\end{array}$ & 5 \\
\hline United States & 11 & incidence & 5 \\
\hline Australia & 9 & information dissemination & 5 \\
\hline research design & 9 & online social networking & 5 \\
\hline risk assessment & 9 & pilot projects & 5 \\
\hline risk-taking & 9 & program evaluation & 5 \\
\hline attitude of health personnel & 8 & retrospective studies & 5 \\
\hline prevalence & 8 & schools & 5 \\
\hline prospective studies & 8 & smartphone & 5 \\
\hline social media/standards & 8 & social environment & 5 \\
\hline $\begin{array}{l}\text { social media/statistics \& numerical } \\
\text { data }\end{array}$ & 8 & treatment outcome & 5 \\
\hline time factors & 8 & United Kingdom & 5 \\
\hline
\end{tabular}

Table 2 reports the frequency of words used in titles in comparison with abstracts. The words "ethics" or "ethical" were retrieved only 33 and 32 times respectively, with the same frequency reported in titles and abstracts. Table 3 shows the frequency of words used in the query related to ethics.

Regarding the conceptual structure of the set of the 368 retrieved articles, Figure 1 shows the co-occurrences of titles words based on the three most frequent words: social (pink network), health (blue network), and study (green network). Different variations of these three words are:

- Social: media, analysis, survey

- Health: online, support, risk, patients, mental, care, cancer

- Study: protocol, qualitative, intervention, trial, randomized, factors, young

Regarding thematic maps of keywords and of titles words (Figures 2 and 3), we identified the most representative clusters according to centrality and density in each quadrant and compare them accordingly (Table 4).

\section{Best Paper Selection}

The 368 retrieved articles were then manually reviewed by section editors, which resulted in 15 articles considered for best paper selection. Elements that were considered in the screening decision include: 1) level of relevance regarding the 2020 Yearbook topic "Ethics in Health Informatics"; 2) whether the study was focused only on patients and consumers; 3 ) nature of the issues addressed; and 4) level of innovative approach.

The selected 15 articles were then presented to a panel of international experts for full paper review and scoring according to the IMIA Yearbook best paper selection process. Only one paper was selected to be the best paper after discussions at a consensus meeting (see Table 5). The description of the study and the main results are described in the Appendix.

The top five studies [6-10] as ranked by the review of external reviewers reported the following ethical concerns when using social media at different phases in a research study.

- Four studies examined public concern [6-9] and one study provides information on professional concern [10]. 
Table 2 Top 10 words and their frequency in titles and abstracts

\begin{tabular}{|l|c|c|c|c|}
\hline Words & Frequency in titles & $\begin{array}{l}\text { Frequency } \\
\text { in abstracts }\end{array}$ & $\begin{array}{c}\text { Ranked according to } \\
\text { frequency in titles (1 } \\
\text { = most frequent) }\end{array}$ & $\begin{array}{c}\text { Ranked according to } \\
\text { frequency in abstracts } \\
\text { (1 = most frequent) }\end{array}$ \\
\hline social & 88 & 755 & 1 & 1 \\
health & 76 & 611 & 2 & 2 \\
study & 68 & 409 & 3 & 3 \\
media & 47 & 357 & 4 & 5 \\
online & 35 & 299 & 5 & 8 \\
analysis & 29 & 206 & 6 & 13 \\
support & 27 & 381 & 7 & 4 \\
risk & 25 & 331 & 8 & 7 \\
intervention & 23 & 177 & 10 & 9 \\
patients & 22 & 288 & & 9 \\
\hline
\end{tabular}

Table 3 Frequency of words found in abstracts

\begin{tabular}{|l|c|c|}
\hline Words & Frequency in abstracts & $\begin{array}{c}\text { Ranked according to frequency in titles } \\
\text { (1 = most frequent })\end{array}$ \\
\hline privacy & 60 & 125 \\
ethics & 33 & 307 \\
ethical & 32 & 324 \\
informed & 23 & 468 \\
protection & 21 & 512 \\
respect & 13 & 843 \\
moral & 10 & 1103 \\
responsibility & 10 & 1103 \\
principle & 7 & 1526 \\
rights & 6 & 1699 \\
bioethics & 2 & 3384 \\
\hline
\end{tabular}

- Two studies analysed the ethical concerns related to recruitment $[6,8]$. First study was selected as best paper [6]. The objective was to ascertain people's attitudes and level of concern about the use of social media monitoring on Twitter for targeted clinical trial recruitment. The expressed attitudes were highly contextual, depending on factors such as the type of disease or health topic and the entity or person who monitored users on Twitter. In the second paper [8], authors tried to determine whether willingness to participate and willingness to share social media data are associated with the type of research team and online recruitment platform. Participants were significantly less likely to participate in federally sponsored or pharmaceutical company research than university-led studies. Authors suggested that researchers may see reduced online research participation and data sharing, particularly for research conducted outside academia.
- One study reported professional opinions on participant engagement [10]. It concluded that engaging participants via social media introduces unique methodological and ethical issues, requiring researchers and ethics committee members to be familiar with the technology and aware of its risks and limitations. Participation in training and access to resources may improve researchers' and ethics committee members' familiarity with social media platforms and ethical conduct.

- One study examined patient perspectives on the risks and benefits of linking existing data sources for research [7]. It concluded that developing methods to link databases to minimise the exposure of unique identifiers may improve patient comfort levels with linking data for research purposes.

- One study explored the ethics of using social media for detecting and monitoring adverse events in research studies [9]. It concluded that there is a wide disparity in attitudes towards research using social media data. Adverse effects are viewed as personal and, therefore, more likely to attract ethical consideration.

\section{Conclusions}

Ethics and health are two professional and research domains intimately connected. Health decision-making process as well as clinical research emphasise the importance of informed consent and ethical principles. Beyond the legislative arsenal framing clinical research and data protection in many countries, the expression of a patient's informed consent is certainly not the only key to create a sustainable trust relationship between the "donor" of data and the data "processor". Findings suggest that most users do not think that monitoring Twitter for clinical trial recruitment constitutes inappropriate surveillance or a violation of privacy. However, further research is needed to identify whether and how views on ethical concerns differed between social media platforms and across populations. 
Staccini et al.

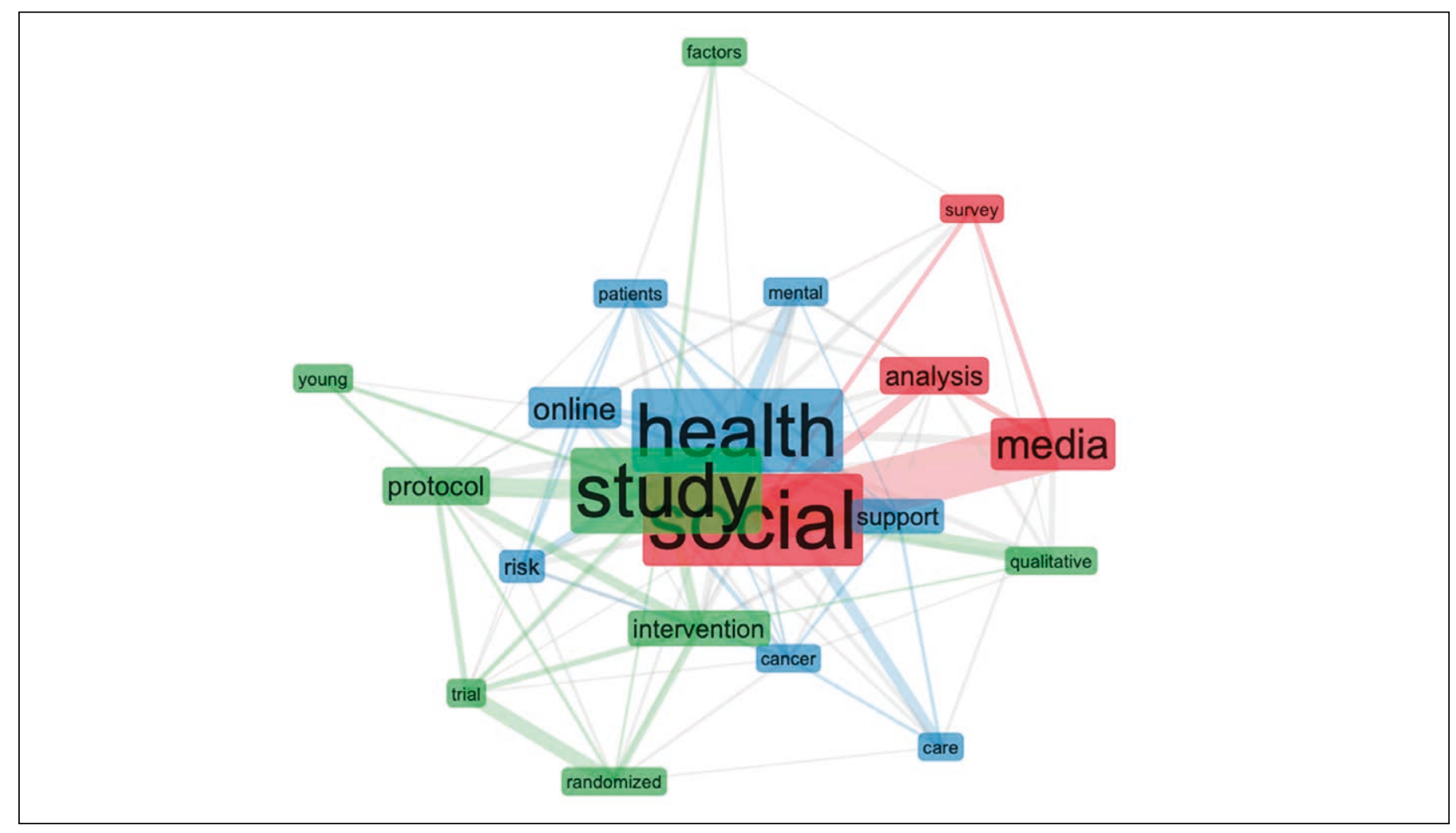

Fig. 1 Co-occurrence network of words used in titles

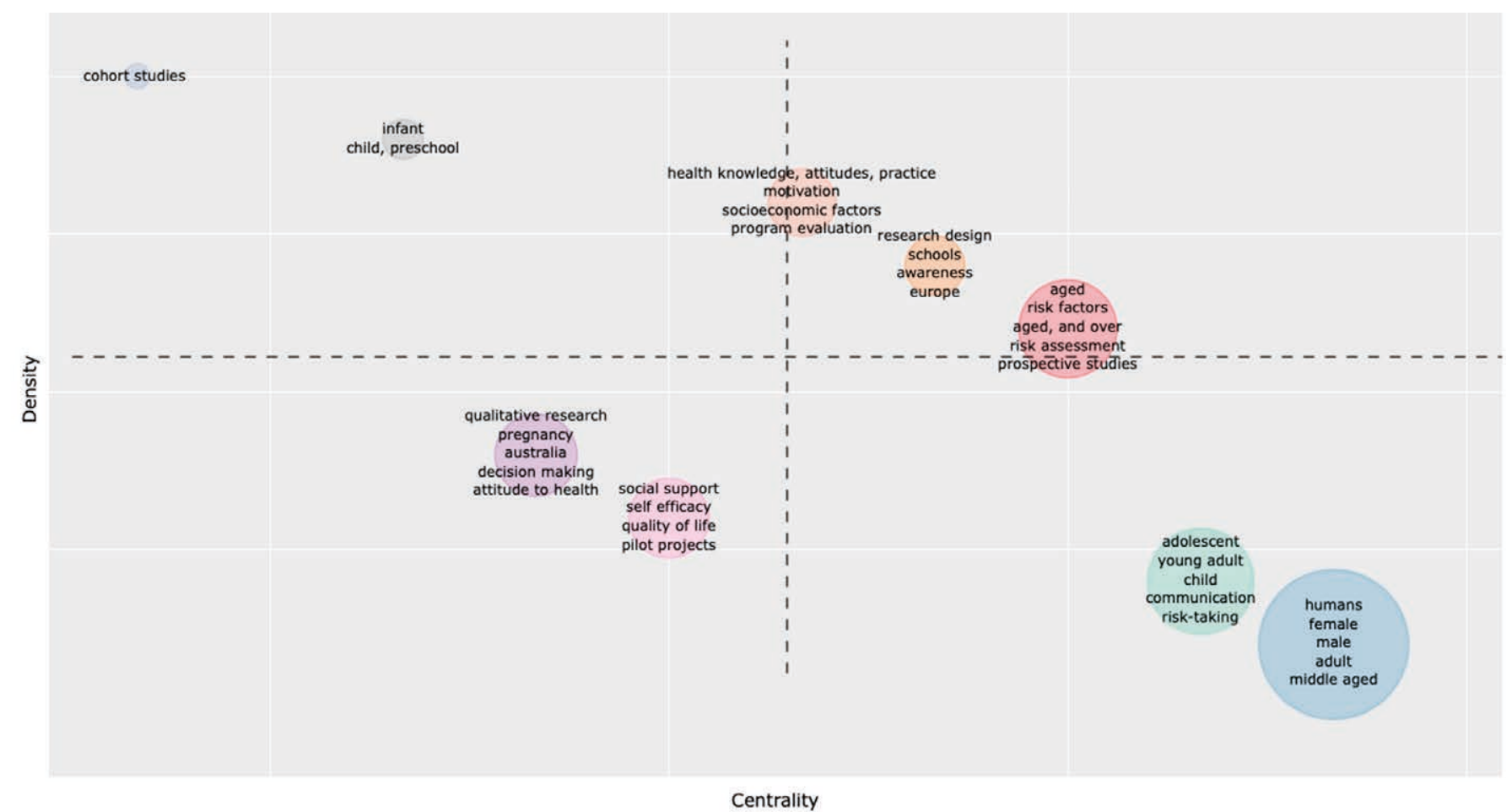

Fig. 2 Thematic map of clusters of words used as keywords

IMIA Yearbook of Medical Informatics 2020 


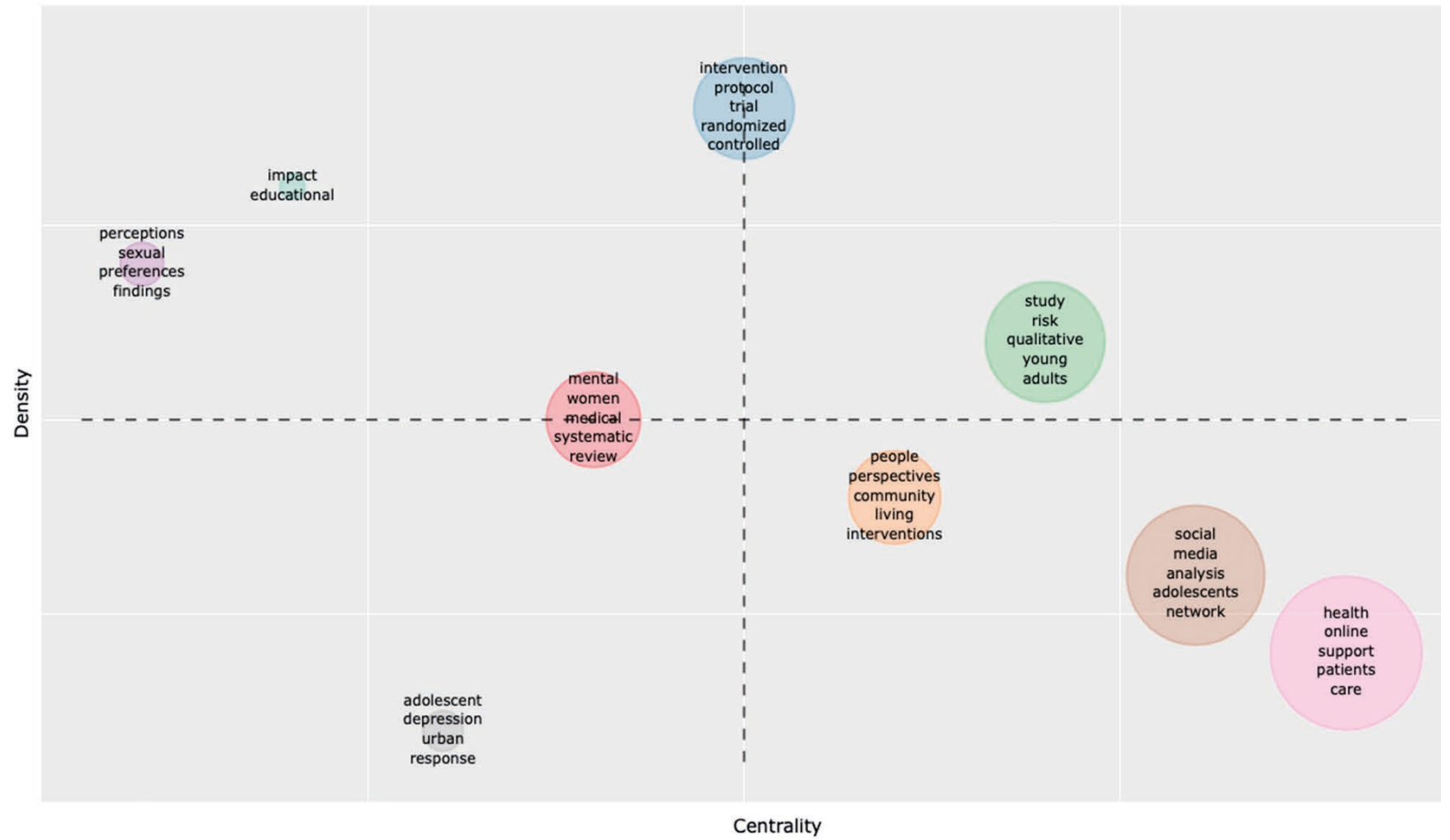

Fig. 3 Thematic map of clusters words found in titles

Table 4 Comparative analysis of thematic maps regarding the locations of clusters

\begin{tabular}{|l|l|l|}
\hline Map location & Keywords map & Titles word map \\
\hline $\begin{array}{l}\text { Upper-right quadrant: motor- } \\
\text { themes }\end{array}$ & $\begin{array}{l}\text { aged, risk factors, aged and over, } \\
\text { risk assessment, prospective studies } \\
\text { humans, female, male, adult, } \\
\text { Lower-right quadrant: basic } \\
\text { themes }\end{array}$ & study, risk, qualitative, young, adults \\
$\begin{array}{l}\text { Lower-left quadrant: emerging or } \\
\text { disappearing themes }\end{array}$ & $\begin{array}{l}\text { qualitative research, Australia, } \\
\text { hecision making, attitude to health }\end{array}$ & $\begin{array}{l}\text { adolescent, depression, urban, } \\
\text { response }\end{array}$ \\
$\begin{array}{l}\text { Upper-left quadrant: very } \\
\text { specialized or niche themes }\end{array}$ & cohort studies & $\begin{array}{l}\text { perceptions, sexual, preferences, } \\
\text { finding }\end{array}$ \\
\hline
\end{tabular}

Table 5 Best paper selection of articles for the IMIA Yearbook of Medical Informatics 2020 in the section 'Consumer Health Informatics and Education'. The articles are listed in alphabetical order of the first author's surname.

\section{Section}

Consumer Health Informatics and Education

- Reuter K, Zhu Y, Angyan P, Le N, Merchant AA, Zimmer M. Public concern about monitoring twitter users and their conversations to recruit for clinical trials: survey study. J Med Internet Res 2019 0ct 30;21(10):e15455.

\section{References}

1. Gelinas L, Pierce R, Winkler S, Cohen IG, Lynch HF, Bierer BE. Using Social Media as a Research Recruitment Tool: Ethical Issues and Recommendations. Am J Bioeth 2017 Mar;17(3):3-14.

2. Gelinas L, Bierer BE. Social Media as an Ethical Tool for Retention in Clinical Trials. Am J Bioeth 2019 Jun;19(6):62-4.

3. Terrasse M, Gorin M, Sisti D. Social Media, E-Health, and Medical Ethics. Hastings Cent Rep 2019 Jan;49(1):24-33.

4. Hammack CM. Ethical Use of Social Media Data: Beyond the Clinical Context. Hastings Cent Rep 2019 Jan;49(1):40-2.

5. Aria, M, Cuccurullo, C. Bibliometrix: An R-tool for comprehensive science mapping analysis. Journal of Informetrics 2017 ;11(4):959-75.

6. Reuter K, Zhu Y, Angyan P, Le N, Merchant AA, Zimmer M. Public Concern About Monitoring Twitter Users and Their Conversations to Recruit for Clinical Trials: Survey Study. J Med Internet Res 2019 Oct 30;21(10):e15455.

7. O'Brien EC, Rodriguez AM, Kum HC, Schanberg LE, Fitz-Randolph M, O`Brien SM, et al. Patient perspectives on the linkage of health data for research: Insights from an online patient community questionnaire. Int J Med Inform 2019 Jul;127:9-17.

8. Pratap A, Allred R, Duffy J, Rivera D, Lee HS, 
Renn BN, et al. Contemporary Views of Research Participant Willingness to Participate and Share Digital Data in Biomedical Research. JAMA Netw Open 2019 Nov 1;2(11):e1915717.

9. Golder S, Scantlebury A, Christmas H. Understanding Public Attitudes Toward Researchers Using Social Media for Detecting and Monitoring Adverse Events Data: Multi Methods Study. J Med
Internet Res 2019 Aug 29;21(8):e7081.

10. Hokke S, Hackworth NJ, Bennetts SK, Nicholson JM, Keyzer P, Lucke J, et al. Ethical Considerations in Using Social Media to Engage Research Participants: Perspectives of Australian Researchers and Ethics Committee Members. J Empir Res Hum Res Ethics 2020 Feb-Apr;15(1-2):12-27.

\section{Correspondence to:}

Pascal Staccini

IRIS Department - Lab RETINES

Université Côte d'Azur - Faculté de Médecine

28 avenue de Valombrose

Nice, France

E-mail: pascal.staccini@univ-cotedazur.fr 


\section{Appendix: Content Summa- ries of Selected Best Paper for the IMIA Yearbook 2020}

Reuter K, Zhu Y, Angyan P, Le N, Merchant AA, Zimmer M

Public concern about monitoring twitter users and their conversations to recruit for clinical trials: survey study

\section{$\mathrm{J}$ Med Internet Res 2019 0ct $30 ; 21(10)$ :e15455}

This study used two online surveys to examine public attitudes of using Twitter to recruit clinical trial participants. While nearly half the survey respondents agreed that social media monitoring constitutes a form of eavesdropping, the authors concluded that most social media users do not think monitoring Twitter for clinical trial recruit- ment constitutes inappropriate surveillance or a violation of privacy. The authors also remind researchers to remain mindful that some participants might find social media monitoring problematic when associated with certain conditions or health topics, and that further research is required to isolate factors that influence the level of concern among social media users across platforms and populations. 